\title{
Eight-component two-fermion equations
}

\author{
Ruth Häckl, Viktor Hund and Hartmut Pilkuhn \\ Institut für Theoretische Teilchenphysik, Universität, D-76128 Karlsruhe, Germany
}

\begin{abstract}
An eight-component formalism is proposed for the relativistic two-fermion problem. In QED, it extends the applicability of the Dirac equation with hyperfine interaction to the positronium case. The use of exact relativistic two-body kinematics entails a CP-invariant spectrum which is symmetric in the total cms energy. It allows the extension of recent $\alpha^{6}$ recoil corrections to the positronium case, and implies new recoil corrections to the fine and hyperfine structures and to the Bethe logarithm.
\end{abstract}

PACS number: 03.65.Pm

\section{INTRODUCTION}

The relativistic two-body problem for two spin-1/2 particles is based on 16-component wave functions which transform as the direct product of two four-component Dirac spinors, $\psi^{(16)} \sim \psi_{1} \otimes \psi_{2}$. For unequal masses $m_{2}>m_{1}$, the equations are simplified by the elimination of the small components of particle 2 and by a subsequent power series expansion about the non-relativistic limit of this particle. One thus obtains an effective Dirac equation for particle 1 , with a hyperfine interaction that contains the Pauli matrices $\boldsymbol{\sigma}_{2}$ of particle 2 . Such an equation has $4 \times 2=8$ components. It is very powerful for hydrogen and muonium 114 . More recently, a non-relativistic quantum-electrodynamics (NRQED) has been elaborated which allows one to eliminate the small components of both particles, which is particularly useful for the equal-mass case, $m_{1}=m_{2}$ as in positronium [0,6]. One thus arrives at an effective Schrödinger equation, in which the Pauli matrices $\boldsymbol{\sigma}_{1}$ and $\boldsymbol{\sigma}_{2}$ produce a fourcomponent spin structure. However, the fact that the power series expansions in $\alpha$ are rapidly converging, does not prevent technical difficulties, presently at the order $\alpha^{6}$ in the binding energies. These $\alpha^{6}$-terms have only been calculated by the above eight-component strategy, and only to first order in $m_{1} / m_{2}$.

In this paper, a different eight-component equation is derived which does not eliminate small components and dispenses with nonrelativistic expansions. It exploits the fact that the chirality operator $\gamma_{1}^{5} \gamma_{2}^{5}$ commutes with the matrix $\gamma_{1}^{0} \gamma_{2}^{0}$ of the parity transformation; a corresponding separation of components does not exist for a single Dirac particle. We begin with the rederivation of an eight-component equation for two free spin- $1 / 2$ particles, in which the spin operator of particle 2 is removed in the cms $\left(\boldsymbol{p}_{1}=-\boldsymbol{p}_{2}=\boldsymbol{p}\right)[7-9]$, by 
means of a new matrix $c$, given in (20) below. The spin dependence in the lab system is generated by a boost, to be discussed in section 5 . The removal matrix $c$ also mixes large and small components, via a Dirac matrix $\beta=\gamma^{0}$. For total cms energy $E$, the result is an effective single-particle Dirac equation, for a free particle of reduced mass $\mu$ and reduced energy $\epsilon$ :

$$
\mu=m_{1} m_{2} / E, \quad \epsilon=\left(E^{2}-m_{1}^{2}-m_{2}^{2}\right) / 2 E, \quad E=m_{1}+m_{2}+E_{b} .
$$

In section 3, the QED Born amplitude for cms scattering will be used to derive the interaction for this equation. The equation with interaction is for two leptons (no anomalous magnetic moments)

$$
(\epsilon-\mu \beta-V) \psi=\gamma_{5}\left(\boldsymbol{\sigma}_{1}-i \boldsymbol{\sigma}^{\times} V / E\right) \boldsymbol{p} \psi, \quad \boldsymbol{\sigma}^{\times}=\boldsymbol{\sigma}_{1} \times \boldsymbol{\sigma}_{2} .
$$

Setting $\gamma_{5} \boldsymbol{\sigma}_{1}=\boldsymbol{\alpha}=\gamma^{0} \boldsymbol{\gamma}$, the equation has the appearance of the usual Dirac equation with hyperfine interaction, particularly as $\boldsymbol{\alpha} \times \boldsymbol{\sigma}_{2} / E$ may be approximated by $\boldsymbol{\alpha} \times \boldsymbol{\sigma}_{2} /\left(m_{1}+m_{2}\right)$ to order $\alpha^{4}$. However, the complete operator $-i \boldsymbol{\alpha} \times \boldsymbol{\sigma}_{2} V \mathbf{p} / E$ contains an antihermitian part which ensures exact relativistic two-body kinematics; for $m_{1}=m_{2}$, it produces the correct spin structure to order $\alpha^{4}$ [9]. A previous derivation from the 16-component DiracBreit equation produced a different hyperfine operator, which is equivalent to the new one only near threshold, $-E_{b} / E \ll 1$. The new hyperfine operator is left invariant by the $c$ transformation. (The previous derivation also had to assume a point Coulomb potential, $V=-Z \alpha / r, \alpha=e^{2}, Z e=$ nuclear charge.)

The relativistic on-shell two-body kinematics has long been well known. One has $\epsilon^{2}-\mu^{2}=$ $E_{1}^{2}-m_{1}^{2}=E_{2}^{2}-m_{2}^{2}=k^{2}$ in the cms, where $k^{2}=-\kappa^{2}$ is negative for bound states, and the asymptotic form $\mathrm{e}^{-\kappa \mathrm{r}}$ of $\psi$ displays $\kappa^{-1}$ as multiple of a Bohr radius. The incorporation of relativistic two-body kinematics results in a spectrum which expresses $E^{2}$ in terms of $m_{1}^{2}$ and $m_{2}^{2}$. The combination $E \mu=m_{1} m_{2}$ is allowed in front of a square root (and possibly also with odd powers of $Z \alpha$, beginning with $Z^{5} \alpha^{5}$ ). Our main nonperturbative result is

$$
E^{2}-m_{1}^{2}-m_{2}^{2}=2 m_{1} m_{2}\left(1+Z^{2} \alpha^{2} / n^{* 2}\right)^{-1 / 2}, \quad n^{*}=n-\beta_{d},
$$

where $n^{*}$ is an effective principal quantum number, and $\beta_{d}$ is a quantum defect. Except for the details of $\beta_{d}$, (3) applies to any combinations of spins. It was first derived for two spinless particles [10], where also its empirical applicability to parapositronium was noticed. It was then extended to the case of one spin-1/2 and one spinless particle [11]. The angular momentum defect, $\beta_{l}=-\delta l=-\left(l^{\prime}-l\right)$, has been discussed for two fermions of arbitrary magnetic moments to order $Z^{4} \alpha^{4}$ [9]. It will be used in section 8 to derive a new "BarkerGlover" term in the fine structure. In section 4, a rather general formula for $Z^{6} \alpha^{6}$ recoil terms will be derived which includes several new effects. In section 6 , a vector potential is included in (2) and evaluated in the dipole approximation. It leads to two additional quantum defects,

$$
n^{*}=n+\delta l-\beta_{B}-\beta^{\prime} \delta_{l 0}
$$

where $\beta_{B}$ is caused by the Bethe logarithm and $\beta^{\prime}$ is an additional quantum defect in sstates. Parts of the Salpeter correction [2] are not included in (41); their mass dependence 
is examined in section 7. Vacuum polarization and nuclear charge distributions are also

discussed in that section. For antiprotonic atoms, vacuum polarization must be included as a part of $V$ for low- $l$-states. A method is proposed which extends the validity of (3) to such states. However, also in cases where (2) must be solved numerically, the $E^{2}$-dependence remains and adds new "recoil" corrections to the binding energies $E_{b}$ of (四).

It may be worth mentioning that the $E^{2}$-dependence of the spectrum is a very general consequence of the CPT-theorem. With the separate validity of $\mathrm{C}, \mathrm{P}$, and $\mathrm{T}$ in $\mathrm{QED}$, one may also say that the $E^{2}$-dependence follows from $\mathrm{C}$-invariance, but in the relativistic case the CP-transformation is slightly more convenient than $\mathrm{C}$ alone. Of course, the states which we calculate are CP-eigenstates only in the case of positronium. Muonium is transformed into antimuonium under CP.

\section{THE FREE EIGHT-COMPONENT EQUATIONS}

Let particles $i(=1,2)$ satisfy the free Dirac equations, in units $\hbar=c=1$ :

$$
\left(i \partial_{i}^{0}-\boldsymbol{\alpha}_{i} \mathbf{p}_{i}-m_{i} \beta_{i}\right) \psi_{i}=0, \quad \boldsymbol{\alpha}_{i}=\gamma_{i}^{5} \boldsymbol{\sigma}_{i}, \quad \beta_{i}=\gamma_{i}^{0}, \quad \partial_{i}^{0}=\partial / \partial t_{i}
$$

The $\boldsymbol{\sigma}_{i}$ are Pauli matrices, and $\gamma_{i}^{5} \beta_{i}+\beta_{i} \gamma_{i}^{5}=0$. The direct product $\psi^{(16)}=\psi_{1} \otimes \psi_{2}$ satisfies both equations (5) and thus also their sum, in which $i \partial_{1}^{0}+i \partial_{2}^{0}$ will be replaced by its eigenvalue $K^{0}$, which is the total lab energy:

$$
\left(K^{0}-\gamma_{1}^{5} \mathbf{p}_{1} \boldsymbol{\sigma}_{1}-\gamma_{2}^{5} \mathbf{p}_{2} \boldsymbol{\sigma}_{2}-m_{1} \beta_{1}-m_{2} \beta_{2}\right) \psi^{(16)}=0
$$

$\psi^{(16)}$ is now divided into two octets $\psi_{L P}$ and $\chi_{L P}$, which have $\gamma_{1}^{5}=\gamma_{2}^{5} \equiv \gamma_{5}$ and $\gamma_{1}^{5}=-\gamma_{2}^{5}=$ $\gamma_{5}$, respectively:

$$
\begin{aligned}
& \left(K^{0}-\gamma_{5} \mathbf{p}_{1} \boldsymbol{\sigma}_{1}-\gamma_{5} \mathbf{p}_{2} \boldsymbol{\sigma}_{2}\right) \psi_{L P}=\left(m_{1} \beta_{1}+m_{2} \beta_{2}\right) \chi_{L P} \\
& \left(K^{0}-\gamma_{5} \mathbf{p}_{1} \boldsymbol{\sigma}_{1}+\gamma_{5} \mathbf{p}_{2} \boldsymbol{\sigma}_{2}\right) \chi_{L P}=\left(m_{1} \beta_{1}+m_{2} \beta_{2}\right) \psi_{L P}
\end{aligned}
$$

The coupling between $\psi_{L P}$ and $\chi_{L P}$ arises because each $\beta_{i}$ reverses the eigenvalue of $\gamma_{i}^{5}$. In the chiral basis, $\gamma_{1}^{5}$ and $\gamma_{2}^{5}$ are diagonal:

$$
\gamma_{i}^{5}=\left(\begin{array}{rr}
1 & 0 \\
0 & -1
\end{array}\right), \beta_{i}=\left(\begin{array}{ll}
0 & 1 \\
1 & 0
\end{array}\right), \psi_{i}=\left(\begin{array}{l}
\psi_{i r} \\
\psi_{i l}
\end{array}\right), \psi_{L P}=\left(\begin{array}{c}
\psi_{r r} \\
\psi_{l l}
\end{array}\right), \chi_{L P}=\left(\begin{array}{c}
\psi_{r l} \\
\psi_{l r}
\end{array}\right)
$$

where the indices $r$ and $l$ (= righthanded, lefthanded) refer to the eigenvalues \pm 1 of $\gamma_{1}^{5}$ and $\gamma_{2}^{5}$. In the parity basis, the $\beta_{i}$ are diagonal, with eigenvalues \pm 1 for the large and small components $g$ and $f$, respectively:

$$
\begin{gathered}
\gamma_{i}^{5}=\left(\begin{array}{ll}
0 & 1 \\
1 & 0
\end{array}\right), \quad \beta_{i}=\left(\begin{array}{rr}
1 & 0 \\
0 & -1
\end{array}\right), \quad \psi_{i}=\left(\begin{array}{l}
\psi_{i g} \\
\psi_{i f}
\end{array}\right), \\
\psi_{L P}=\left(\begin{array}{l}
\psi_{g g}+\psi_{f f} \\
\psi_{f g}+\psi_{g f}
\end{array}\right), \quad \chi_{L P}=\left(\begin{array}{c}
\psi_{g g}-\psi_{f f} \\
\psi_{f g}-\psi_{g f}
\end{array}\right) .
\end{gathered}
$$


In this eight-component space, we also define a new matrix $\beta$ :

$$
\beta=\beta_{1} \beta_{2}=\left(\begin{array}{rr}
1 & 0 \\
0 & -1
\end{array}\right), \quad \beta \gamma_{5}+\gamma_{5} \beta=0
$$

(unit matrices are suppressed). Whereas $\beta_{i}$ and $\gamma_{i}^{5}$ do not commute, $\beta$ does commute with $\gamma_{1}^{5} \gamma_{2}^{5}$, and both operators are diagonal in the representation (11). As Lorentz transformations commute with $\gamma_{i}^{5}$ and parity transformations commute with $\beta_{i}$, the decomposition of $\psi^{(16)}$ into $\psi_{L P}$ and $\chi_{L P}$ is invariant under the extended group of Lorentz $(L)$ and parity $(P)$ transformations. In the following $\chi_{L P}$ will be eliminated. We introduce a compact notation,

$$
p_{ \pm}=\mathbf{p}_{1} \boldsymbol{\sigma}_{1} \pm \mathbf{p}_{2} \boldsymbol{\sigma}_{2}, \quad m_{ \pm}=m_{2} \pm \beta m_{1},
$$

and observe $\beta_{2} \psi_{L P}=\chi_{L P}, \quad \beta_{2} \chi_{L P}=\psi_{L P}$ in the basis (11), such that we may effectively put $\beta_{2}=1$ and $\beta_{1}=\beta$ in (7), (\$) in this basis:

$$
\left(K^{0}-\gamma_{5} p_{+}\right) \psi_{L P}=m_{+} \chi_{L P}, \quad\left(K^{0}-\gamma_{5} p_{-}\right) \chi_{L P}=m_{+} \psi_{L P} .
$$

Using the first equation for the elimination of $\chi_{L P}$, one obtains for the second one

$$
\left(K^{0}-\gamma_{5} p_{-}\right)\left(m_{+}\right)^{-1}\left(K^{0}-\gamma_{5} p_{+}\right) \psi_{L P}=m_{+} \psi_{L P} .
$$

Multiplying this equation by $m_{+}$and using

$$
m_{+} m_{-}=m_{2}^{2}-m_{1}^{2}, \quad m_{+} \gamma_{5}=\gamma_{5} m_{-}
$$

one arrives at the following equation

$$
\mathcal{K}_{0} \psi_{L P}=0, \quad \mathcal{K}_{0}=\left(K^{0}-\gamma_{5} p_{-} m_{-} / m_{+}\right)\left(K^{0}-\gamma_{5} p_{+}\right)-m_{+}^{2} .
$$

Equations (14) or (17) can be Lorentz transformed to the cms, where one has $K^{0}=$ $E, p_{-} p_{+}=p_{1}^{2}-p_{2}^{2}=0$. The constants of (17) are combined into $E^{2}-m_{1}^{2}-m_{2}^{2}=2 E \epsilon$, with $\epsilon$ defined by (11):

$$
\left[2 E \epsilon-E \gamma_{5}\left(\mathbf{p} \boldsymbol{\sigma} m_{-} / m_{+}+\mathbf{p} \Delta \boldsymbol{\sigma}\right)\right] \psi_{L P}=2 m_{1} m_{2} \beta \psi_{L P}, \quad \boldsymbol{\sigma}=\boldsymbol{\sigma}_{1}+\boldsymbol{\sigma}_{2}, \quad \Delta \boldsymbol{\sigma}=\boldsymbol{\sigma}_{1}-\boldsymbol{\sigma}_{2},
$$

where $m_{1} m_{2}$ may also be written as $E \mu$ according to (1). If $\mathbf{p} \boldsymbol{\sigma}$ were present without the factor $m_{-} / m_{+}$, one would use $\mathbf{p} \boldsymbol{\sigma}+\mathbf{p} \Delta \boldsymbol{\sigma}=2 \mathbf{p} \boldsymbol{\sigma}_{1}$, and equation (18) would be identical with equation (2) for $V=0$, multiplied by $2 E$.

It is in fact possible to remove $m_{-} / m_{+}$from (18), by a transformation

$$
\psi_{L P}=c \psi, \quad c^{-1} \gamma_{5}=\gamma_{5} c, \quad c \boldsymbol{\sigma} c=\boldsymbol{\sigma} m_{+} / m_{-}, \quad c \Delta \boldsymbol{\sigma} c=\Delta \boldsymbol{\sigma}
$$

Explicit forms of $c$ are

$$
c=\left(m_{+} m_{-}\right)^{-1 / 2}\left[m_{2}+\frac{1}{2} m_{1} \beta\left(1+\boldsymbol{\sigma}_{1} \boldsymbol{\sigma}_{2}\right)\right]=\left(m_{+} m_{-}\right)^{-1 / 2}\left(m_{+}-2 m_{1} \Lambda_{s}\right),
$$

where $\Lambda_{s}=\left(1-\boldsymbol{\sigma}_{1} \boldsymbol{\sigma}_{2}\right) / 4$ is the projector on singlet spin states. To verify (19), one notices $\Lambda_{s} \boldsymbol{\sigma}=\boldsymbol{\sigma} \Lambda_{s}=0$. In summary, the 16-component equation (6) is now transformed into a single free-Dirac equation $\left(\epsilon-\mu \beta-\gamma_{5} \boldsymbol{\sigma}_{1} \mathbf{p}\right) \psi_{\text {free }}=0$, with no trace of the spin operators of particle 2 . In the next section, it will be seen that the interaction for this equation contains no mass factors at all. 


\section{THE ONE-PHOTON EXCHANGE INTERACTION}

A connection between bound states and perturbative $Q E D$ rests on the $S$-matrix $S=$ $1+i T$ and the Born series for the $T$-matrix, $T=T^{(1)}+T^{(2)}+\ldots$. When this series is summed by appropriate differential or integral equations, the bound states appear as poles of $T$. In detail, one takes plane waves, $\psi_{i}=u_{i} e^{i \phi_{i}}$ with $\phi_{i}=\mathbf{k}_{i} \mathbf{r}_{i}-E_{i} t$ for the initial states, $\phi_{i}^{\prime}=\mathbf{k}_{i}^{\prime} \mathbf{r}_{i}-E_{i}^{\prime} t$ for the final states and extracts the resulting energy-momentum conserving $\delta$-function from the $T$-matrix elements, $S_{i f}=i(2 \pi)^{4} \delta\left(E-E^{\prime}\right) \delta\left(\mathbf{k}_{1}+\mathbf{k}_{2}-\mathbf{k}_{1}^{\prime}-\mathbf{k}_{2}^{\prime}\right) T_{i f}$. In analogy, we put $\psi_{L P}=v e^{i \phi}, \chi_{L P}=w e^{i \phi}$ with $\phi=\phi_{1}+\phi_{2}$. The first Born approximation to $T_{i f}^{(1)}$ of the matrix elements $T_{i f}$ is (with $q_{1}=-e, q_{2}=Z e, e^{2}=\alpha$ )

$$
T_{i f}^{(1)}=\frac{4 \pi Z \alpha}{t} u_{1}^{\prime \dagger} u_{2}^{\prime \dagger}\left(1-\boldsymbol{\alpha}_{1} \boldsymbol{\alpha}_{2}\right) u_{1} u_{2}=\frac{4 \pi Z \alpha}{t}\left[v^{\prime \dagger}\left(1-\boldsymbol{\sigma}_{1} \boldsymbol{\sigma}_{2}\right) v+w^{\prime \dagger}\left(1+\boldsymbol{\sigma}_{1} \boldsymbol{\sigma}_{2}\right) w\right]
$$

with $t=q^{0^{2}}-\mathbf{q}^{2}, q^{0}=K_{1}^{0}-K_{1}^{0^{\prime}}, \mathbf{q}=\mathbf{k}_{1}-\mathbf{k}_{1}^{\prime}$. Using (14) for the elimination of $v^{\prime \dagger}$ in one term and of $w$ in the other, $T_{i f}^{(1)}$ is expressed in terms of an $8 \times 8$-matrix $M$ :

$$
w=\frac{1}{m_{+}}\left(K^{0}-\gamma_{5} k_{+}\right) v, \quad v^{\prime \dagger}=w^{\prime \dagger}\left(K^{0^{\prime}}-\gamma_{5} k_{-}^{\prime}\right) \frac{1}{m_{+}}, \quad T_{i f}^{(1)}=w^{\prime \dagger} M v .
$$

In the differential equation approach based on (5), the potential $V$ is the Fourier transform of $T^{(1)}$, apart from corrections from the hermitian part of $T^{(2)}$ [12]. Unitarity $S^{\dagger} S=1$ implies $T^{(1)^{\dagger}}=T^{(1)}$ and thus $V=V^{\dagger}$, i.e. a hermitian potential. However, the asymmetric form $w^{\prime \dagger} M v$ implies a non-hermitian interaction $\mathcal{K}_{I}$ in our differential equation $\left(\mathcal{K}_{0}+\mathcal{K}_{I}\right) \psi=0$. The simultaneous validity of $\left(\mathcal{K}_{0}+\mathcal{K}_{I}^{\dagger}\right) \chi=0$ guarantees real eigenvalues, though. The ordinary Dirac equation fails to order $Z^{4} \alpha^{4}$ in the states with $l=f(l=$ orbital, $f=$ total angular momentum), due to hyperfine mixing.

We now restrict ourselves to the cms, $\mathbf{k}_{1}+\mathbf{k}_{2}=\mathbf{k}_{1}^{\prime}+\mathbf{k}_{2}^{\prime}=0$ and call $\mathbf{k}_{1}=\mathbf{k}, \mathbf{p}_{1}=\mathbf{p}=$ $-i \boldsymbol{\nabla}$. The total phase $\phi$ contains $\mathbf{k}\left(\mathbf{r}_{1}-\mathbf{r}_{2}\right)=\mathbf{k r}$, such that one has $\mathbf{p}_{2}=-\mathbf{p}$. Moreover, $K^{0}=K^{0^{\prime}}=E$. To order $Z^{4} \alpha^{4}$, one also has $q^{0}=0, t=-\mathbf{q}^{2}$ :

$$
-\mathbf{q}^{2} M / 4 \pi Z \alpha=\left(E-\gamma_{5} k_{-}^{\prime}\right) m_{+}^{-1}\left(1-\boldsymbol{\sigma}_{1} \boldsymbol{\sigma}_{2}\right)+m_{+}^{-1}\left(1+\boldsymbol{\sigma}_{1} \boldsymbol{\sigma}_{2}\right)\left(E-\gamma_{5} k_{+}\right) .
$$

With $k_{-}^{\prime}=\mathbf{k}^{\prime} \boldsymbol{\sigma}$, one has $k_{-}^{\prime}\left(1-\boldsymbol{\sigma}_{1} \boldsymbol{\sigma}_{2}\right)=4 \mathbf{k}^{\prime} \boldsymbol{\sigma} \Lambda_{s}=0$, as $1-\boldsymbol{\sigma}_{1} \boldsymbol{\sigma}_{2}$ vanishes for triplet states, while $\boldsymbol{\sigma}$ annihilates the singlet state. Consequently,

$$
-\mathbf{q}^{2} M / 4 \pi Z \alpha=m_{+}^{-1}\left[2 E-\gamma_{5}\left(1+\boldsymbol{\sigma}_{1} \boldsymbol{\sigma}_{2}\right) \mathbf{k} \Delta \boldsymbol{\sigma}\right]=2 m_{+}^{-1}\left(E-i \gamma_{5} \mathbf{k} \boldsymbol{\sigma}^{\times}\right) .
$$

Combining now $m_{+}^{-1}$ with $w^{++}$, the Fourier transform of $m_{+} M$ will be called $\mathcal{K}_{I}$ :

$$
\mathcal{K}_{I}=E V-i \gamma_{5} V \mathbf{p} \boldsymbol{\sigma}^{\times}, \quad V=-Z \alpha / r .
$$

This is to be added to $\mathcal{K}_{0}$ (17) and used in (18). To arrive at the form (2) of the differential equation, $\mathcal{K}_{I}$ must be replaced by $c^{-1} \mathcal{K}_{I} c$ according to (19). Fortunately, one finds

$$
c \boldsymbol{\sigma}^{\times} c=\boldsymbol{\sigma}^{\times}, \quad c^{-1} \mathcal{K}_{I} c=\mathcal{K}_{I},
$$

such that the same operator appears in fact in (2). To order $Z^{4} \alpha^{4}$ in $E, \mathcal{K}_{I}$ is equivalent to the operator $\mathcal{K}_{I B}=E V-\gamma_{5}\left(i \boldsymbol{\sigma}^{\times}-\boldsymbol{\sigma}_{2}\right)[V, \mathbf{p}]$ of the Dirac-Breit approach [8,9], the transformation being 


$$
\psi_{B}=e^{\boldsymbol{\sigma}_{1} \boldsymbol{\sigma}_{2} V / 2 E} \psi,
$$

after the transformation $c$ (notice $c^{-1} \mathcal{K}_{I B} c \neq \mathcal{K}_{I B}$ ).

For the orders $Z^{5} \alpha^{5}$ and $Z^{6} \alpha^{6}$, the condition $q^{0}=0$ should be replaced by the current conservation conditions, $q_{\mu} J_{i}^{\mu}=0(i=1,2)$. This produces the spin operator $\boldsymbol{\sigma}_{1}^{\perp} \boldsymbol{\sigma}_{2}^{\perp}=$ $\boldsymbol{\sigma}_{1} \boldsymbol{\sigma}_{2}-\left(\boldsymbol{\sigma}_{1} \boldsymbol{q}\right)\left(\boldsymbol{\sigma}_{2} \boldsymbol{q}\right) / \boldsymbol{q}^{2}$, which is equivalent to using the Coulomb gauge. However, we have not yet performed this calculation. The new $Z^{6} \alpha^{6}$-terms of (42) below result from the use of relativistic kinematics in (2).

\section{SOLVING THE NEW EQUATION NONPERTURBATIVELY}

The total angular momentum $\mathbf{F}=\mathbf{L}+\left(\boldsymbol{\sigma}_{1}+\boldsymbol{\sigma}_{2}\right) / 2=\mathbf{J}_{1}+\boldsymbol{\sigma}_{2} / 2$ is conserved. All eight components of $\psi$ have the same eigenvalues $f(f+1)=F^{2}$ of $\mathbf{F}^{2}$ and $m_{f}$ of $F_{z}$; factors $1 / r$ and $i / r$ are extracted from $\psi_{g}$ and $\psi_{f}$ as usual:

$$
\begin{gathered}
\psi_{g k}=\chi_{k}^{f m_{f}} \frac{1}{r} u_{g k}(r), \quad \psi_{f k}=\chi_{k}^{f m_{f}} \frac{i}{r} u_{f k}(r), \\
(\epsilon-\mu-V) u_{g}=i \widetilde{\pi} u_{f}, \quad(\epsilon+\mu-V) u_{f}=-i \widetilde{\pi} u_{g}, \quad \widetilde{\pi}=\left(\boldsymbol{\sigma}_{1}-i \boldsymbol{\sigma}^{\times} V / E\right) r \mathbf{p} / r .
\end{gathered}
$$

The index $k$ assumes the four values $k=s=(f, 0)$ (singlet), $k=1=(f, 1)$ (triplet with orbital angular momentum $l=f$ ) and $k=+, k=-$ (triplets with $l \neq f$ and eigenvalues $\mp 1$ of $\left.\sigma_{1 r} \sigma_{2 r}=\boldsymbol{\sigma}_{1} \hat{\boldsymbol{r}} \boldsymbol{\sigma}_{2} \hat{\boldsymbol{r}}\right)[8]$. We arrange the radial wave functions as follows:

$$
u_{g}(r)=\left(\begin{array}{c}
u_{g+} \\
u_{g-} \\
u_{g 1} \\
u_{g s}
\end{array}\right), \quad u_{f}(r)=\left(\begin{array}{l}
u_{f+} \\
u_{f-} \\
u_{f 1} \\
u_{f s}
\end{array}\right), \quad \frac{\pi}{i}=\left(\begin{array}{cccc}
0 & 0 & -F / r & f_{-} \partial_{-} \\
0 & 0 & \partial_{r} & -f_{-} F / r \\
F / r & \partial_{r} & 0 & 0 \\
f_{+} \partial_{+} & f_{+} F / r & 0 & 0
\end{array}\right),
$$

with $\partial_{ \pm}=\partial_{r} \pm 1 / r, f_{ \pm}=1 \pm 2 V / E$. The states $k= \pm$ with $l=f \pm 1$ have $j_{1}=j_{2}=f \pm 1 / 2$ to order $\alpha^{2}$. An analytic solution of the equations is obtained when the hyperfine operator is replaced by an equivalent $r^{-2}$-operator. At this level of precision, the equation becomes equivalent to the one derived from the Dirac-Breit equation [8.9], where the replacement was achieved by the substitution $r=r^{\prime}-Z \alpha a / 2 \mu$ (see section 8 for $a$ ).

$$
\left[\epsilon^{2}-\mu^{2}-2 \epsilon V-\tilde{L}^{2} / r^{2}+\partial_{r}^{2}\right] u=0 .
$$

$\tilde{L}^{2}=L^{2}-Z^{2} \alpha^{2}(1+a)$ comprises all $r^{-2}$-operators. Its eigenvalues will be denoted by $l^{\prime}\left(l^{\prime}+1\right)$, to profit from the analogy with the Schrödinger equation. Defining moreover

$$
\epsilon^{2}-\mu^{2}=-\kappa^{2}, \quad z=-2 i k r=2 \kappa r, \quad \alpha_{Z}=Z \alpha, \quad \alpha_{Z} \epsilon / \kappa \equiv n^{*},
$$

and $u=e^{-z / 2} z^{l^{\prime}} F(z)$, the equation becomes as usual an equation for the confluent hypergeometric function $F$,

$$
\left[l^{\prime}+1-z \partial_{z}^{2}-\left(2 l^{\prime}+2-z\right) \partial_{z}\right] F=n^{*} F
$$


For unbound states, $\eta=-i n^{*}$ is called the Sommerfeld parameter. For bound states, $n^{*}-l^{\prime}-1$ must be a non-negative integer $n_{r}$. With $l^{\prime}$ near an integer $l$, one defines

$$
\delta l=l^{\prime}-l, \quad n^{*}=n_{r}+l^{\prime}+1=n+\delta l,
$$

where $n=n_{r}+l+1$ is the principal quantum number and $\delta l$ is always negative. All four eigenvalues $\lambda$ of $\tilde{L}^{2}-F^{2}+\alpha_{Z}^{2}$ are given by

$$
\lambda^{2}\left[\lambda^{2}-2 \lambda-4\left(F^{2}-\alpha_{Z}^{2} \epsilon / E\right)+2 \alpha_{Z}^{2}\right]-2 \alpha_{Z}^{2} \lambda-\alpha_{Z}^{4}(1-4 \epsilon / E)=0 .
$$

It contains the Dirac eigenvalues with recoil-corrected hyperfine structure and hyperfine mixing near both static limits [9]. For equal masses, the equation is reliable only to order $\alpha^{2}$, but for $l=f$ we nevertheless quote the result for $\delta l$ to order $\alpha^{4}$. With $1-4 \epsilon / E=\alpha^{2} / 4 n^{2}$, one solution of (35) is $\lambda=-\alpha^{4} / 8 n^{2}$. It belongs to parapositronium, as we shall see. The value of $l^{\prime}$ follows from $l^{\prime}+\frac{1}{2}=\sqrt{F^{2}+1 / 4+\lambda-\alpha^{2}}=\sqrt{\left(l+\frac{1}{2}\right)^{2}+\lambda-\alpha^{2}}$,

$$
-\delta l_{\text {para }}=\frac{\alpha^{2}}{2 l+1}+\frac{\alpha^{4}}{(2 l+1)^{3}}+\frac{\alpha^{4}}{8 n^{2}(2 l+1)} \text {. }
$$

For orthopositronium with $l=f$, one factor $\lambda$ is divided off and $\lambda^{3}$ can be neglected to order $\alpha^{4}$ :

$$
-\delta l_{\text {ortho }}(l=f)=\frac{\alpha^{2}}{2 l+1}\left[1-\frac{1}{2 F^{2}}-\frac{\alpha^{2}}{2 F^{6}}-\alpha^{2}\left(\frac{1-1 / 2 F^{2}}{2 l+1}\right)^{2}\right]-\frac{\alpha^{4}}{8 n^{2}(2 l+1)} .
$$

We now come to our main point, namely the calculation of $E^{2}$. From (32) and the definition (回) of $\mu$ and $\epsilon$, one finds (3). Expansion of the square root leads to a more practical series, which to order $\alpha_{Z}^{8}$ is

$$
\frac{E^{2}-m^{2}}{m_{1} m_{2}}=-\frac{\alpha_{Z}^{2}}{n^{* 2}}\left[1-\frac{3}{4} \frac{\alpha_{Z}^{2}}{n^{* 2}}+\frac{5}{8} \frac{\alpha_{Z}^{4}}{n^{* 4}}\left(1-\frac{7}{8} \frac{\alpha_{Z}^{2}}{n^{* 2}}\right)\right], \quad m=m_{1}+m_{2} .
$$

Insertion of $n^{*}=n+\delta l$ yields, to order $\alpha_{Z}^{6}$,

$$
\frac{E^{2}-m^{2}}{m_{1} m_{2}}=-\frac{\alpha_{Z}^{2}}{n^{2}}\left(1+\alpha_{Z}^{2} \frac{b}{n}\right), \quad b=-\frac{2 \delta l}{\alpha_{Z}^{2}}-\frac{3}{4 n}+\frac{\alpha_{Z}^{2}}{n}\left(\frac{5}{8 n^{2}}+3 \frac{\delta l}{\alpha_{Z}^{2} n}+3 \frac{\delta l^{2}}{\alpha_{Z}^{4}}\right) .
$$

This expression is still quite compact, in view of the fact that $\delta l$ contains both fine and hyperfine interactions. The result for $E$ is, again to order $\alpha_{Z}^{6}$

$$
\begin{aligned}
E=\left(m^{2}-m_{1} m_{2} \alpha_{Z}^{2}\left(1+\alpha_{Z}^{2} b / n\right) / n^{2}\right)^{1 / 2} & =m-\mu_{n r} \alpha_{Z}^{2}\left(1+\alpha_{Z}^{2} b / n\right) / 2 n^{2} \\
& -\mu_{n r}^{2} \alpha_{Z}^{4}\left(1+\alpha_{Z}^{2} b / n\right)^{2} / 8 n^{4} m-\mu_{n r}^{3} \alpha_{Z}^{6} / 16 n^{6} m^{2},
\end{aligned}
$$

where $\mu_{n r}=m_{1} m_{2} / m$ is the non-relativistic reduced mass, and the expression (39) for $b$ remains to be inserted. The third term of (40), with the approximation $b=0$, is known as the Bechert-Meixner recoil correction $[8]$. To order $\alpha_{Z}^{6}, b$ may be approximated by $-2 \delta l / \alpha_{Z}^{2}-$ $3 / 4 n$. For comparison with the literature [13,4], we split the reduced mass $\mu_{n r}$ in a rather unusual way, 


$$
\mu_{n r}=\mu_{n r}\left(1+\mu_{n r} / m\right)-\mu_{n r}^{2} / m \approx m_{1}^{2}\left(1-m_{1}^{2} / m^{2}\right)-\mu_{n r}^{2} / m
$$

In the order $\alpha_{Z}^{6}$, we may then combine all contributions from (40) with that of an additional operator $\sim L^{2} / r^{4}$ [13], which makes the expression complete for $l>0$ :

$$
\Delta E\left(\alpha_{Z}^{6}\right)=\frac{\mu_{n r}^{2} \alpha_{Z}^{6}}{2 m n^{6}}\left(\frac{4 n \delta l}{\alpha_{Z}^{2}}+1-\frac{\mu_{n r}}{8 m}\right)+\frac{\alpha_{Z}^{2}}{2 \mu_{n r}^{2} m}\left\langle\frac{L^{2}}{r^{4}}\right\rangle .
$$

The last contribution has been symmetrized in the masses, and the expectation value $\left\langle r^{-4}\right\rangle$ refers to the solution of the Schrödinger equation with reduced mass. This operator has been calculated from two-photon exchange, but the order of exchange may be gauge-dependent. Insertion of the Dirac quantum defect

$$
\delta l_{D} / \alpha_{Z}^{2}=\left(\gamma-j-\frac{1}{2}\right) / \alpha_{Z}^{2} \approx-\left[1+\alpha_{Z}^{2} /(2 j+1)^{2}\right] /(2 j+1)
$$

reproduces the known result [13]. Equation (42) generalizes this result to arbitrary masses and hyperfine interactions (section 8).

However, we would like to advocate the direct use of formula (39) for $\left(E^{2}-m^{2}\right) / m_{1} m_{2}$, because it is more compact for calculations and less mass-dependent for measurements.

\section{BOOSTS AND COORDINATE TRANSFORMATIONS}

Lorentz transformations can be constructed directly for $\psi$ and $\psi_{L P}$, but it is more convenient to use the known transformations of the single free-particle spinors $\psi_{1}$ and $\psi_{2}$. We only need the boosts from the cms to the lab system $(l)$, where the system has a total four-momentum $K^{\mu}$ :

$$
\begin{gathered}
\psi_{i, l}=A_{i} \psi_{i, c m}, \quad A_{i}=\left(\gamma+\gamma_{i}^{5} \widehat{\boldsymbol{K}} \boldsymbol{\sigma}_{i}\right)^{1 / 2}=(2 \gamma+2)^{-1 / 2}\left(\gamma+1+\gamma_{i}^{5} \widehat{\boldsymbol{K}} \boldsymbol{\sigma}_{i}\right), \\
\widehat{\boldsymbol{K}}=\boldsymbol{K} / E, \quad \gamma=K^{0} / E=\left(1+\hat{K}^{2}\right)^{1 / 2} .
\end{gathered}
$$

We also take the $z$-axis along $\boldsymbol{K}, \widehat{\boldsymbol{K}} \boldsymbol{\sigma}_{i}=\hat{K} \sigma_{i z}$. Suppressing the index $L P$, the eightcomponent lab spinor is

$$
\begin{gathered}
\psi_{l}=A \psi_{c m}, \quad A=\left(\gamma+\gamma_{5} \hat{K} \sigma_{2 z}\right)^{1 / 2}\left(\gamma+\gamma_{5} \hat{K} \sigma_{1 z}\right)^{1 / 2}=\left(1+\frac{1}{2} \hat{K}^{2} \sigma_{z}^{2}+\hat{K} \sigma_{z} \gamma \gamma_{5}\right)^{1 / 2} \\
A=\gamma+\frac{1}{2} \hat{K} \gamma_{5} \sigma_{z}-\frac{1}{2} \hat{K}^{2} \Delta \sigma_{z}^{2} /(2 \gamma+2)
\end{gathered}
$$

(in checking (47) by squaring of (46), use $\sigma_{z} \Delta \sigma_{z}=0,\left(\Delta \sigma_{z}\right)^{2}=2\left(1-\sigma_{1 z} \sigma_{2 z}\right),\left(\Delta \sigma_{z}\right)^{4}=$ $4\left(\Delta \sigma_{z}\right)^{2}$ and $\left.\hat{K}^{4}=\hat{K}^{2}\left(\gamma^{2}-1\right)\right)$. The boost $\bar{A}$ for $\chi$ follows from $A$ by replacing $\gamma_{5} \boldsymbol{\sigma}_{2}$ by $-\gamma_{5} \sigma_{2}$ :

$$
\chi_{l}=\bar{A} \chi_{c m}, \quad \bar{A}=\gamma+\frac{1}{2} \hat{K} \gamma_{5} \Delta \sigma_{z}-\frac{1}{2} \hat{K}^{2} \sigma_{z}^{2} /(2 \gamma+2)
$$

The inverse boost has $\boldsymbol{K}$ replaced by $-\boldsymbol{K}$, which is equivalent to a sign change of $\gamma_{5}$ : 


$$
A \beta=\beta A^{-1}, \quad \bar{A} \beta=\beta \bar{A}^{-1} .
$$

Insertion of (19), $\psi_{c m}=c \psi$ gives in the lab system $\psi_{l}=A c \psi$, and for the $c$-transformed $\psi_{l}$ :

$$
\begin{gathered}
\psi_{l c}=c^{-1} \psi_{l}=c^{-1} A c \psi=A_{c} \psi, \quad A_{c}=c^{-1} A c, \\
A_{c}=\gamma+\frac{1}{2} \gamma_{5} \hat{K} \sigma_{z} m_{+} / m_{-}-\frac{1}{2} \hat{K}^{2} \Delta \sigma_{z}^{2} /(2 \gamma+2) .
\end{gathered}
$$

The corresponding boost for $\chi$, on the other hand, has a factor $m_{+}$extracted:

$$
\bar{A}_{c}=m_{+}^{-1} A m_{+}=\gamma+\frac{1}{2} \gamma_{5} \hat{K} \Delta \sigma_{z} m_{+} / m_{-}-\frac{1}{2} \hat{K}^{2} \sigma_{z}^{2} /(2 \gamma+2) .
$$

The desired boosts for $\psi$ and $\chi$ are $A_{c}$ and $\bar{A}_{c}$, respectively. They are needed for the construction of Dirac-Breit equations in the presence of external potentials.

In a covariant treatment, the interaction between two particles at distance $\boldsymbol{r}_{l}=\boldsymbol{r}_{1}-\boldsymbol{r}_{2}$ depends also on a time difference $x^{0}=t_{1}-t_{2}$, such that $x^{\mu}=\left(x^{0}, \boldsymbol{r}_{l}\right)$ is a four-vector. A second independent four-vector $X^{\mu}$ is defined such that $P^{\mu}=i \partial_{X}^{\mu}$ becomes the total four-momentum $p_{1}^{\mu}+p_{2}^{\mu}$, which is conserved:

$$
\begin{gathered}
x^{\mu}=x_{1}^{\mu}-x_{2}^{\mu}, \quad X^{\mu}=\hat{E}_{1} x_{1}^{\mu}+\hat{E}_{2} x_{2}^{\mu}, \quad p_{1}^{\mu}=p^{\mu}+\hat{E}_{1} P^{\mu}, \quad p_{2}^{\mu}=-p^{\mu}+\hat{E}_{2} P^{\mu}, \\
\hat{E}_{1}+\hat{E}_{2}=1, \quad P^{\mu}=p_{1}^{\mu}+p_{2}^{\mu}, \quad P^{\mu} \psi=K^{\mu} \psi .
\end{gathered}
$$

The as yet open value of $\hat{E}_{1}-\hat{E}_{2}$ is chosen such that $p^{0}=i \partial / \partial x^{0}$ vanishes at asymptotic distances in the cms where particles 1 and 2 are on their mass shells, $E_{1}^{2}-k^{2}=m_{1}^{2}, E_{2}^{2}-k^{2}=$ $m_{2}^{2}$, i.e. $E_{2}^{2}-E_{1}^{2}=m_{2}^{2}-m_{1}^{2}=m_{+} m_{-}$. Using in addition $E_{1}+E_{2}=E$, one obtains

$$
E_{1}=\left(E^{2}-m_{+} m_{-}\right) / 2 E, \quad E_{2}=\left(E^{2}+m_{+} m_{-}\right) / 2 E .
$$

Extracting now from (53)

$$
p^{\mu}=\hat{E}_{2} p_{1}^{\mu}-\hat{E}_{1} p_{2}^{\mu}
$$

and inserting the asymptotic values $E_{i}$ of $p_{i}^{0}$ in the cms, one finds that $p^{0}$ vanishes here for

$$
\hat{E}_{i}=E_{i} / E=\frac{1}{2}\left(1 \mp \hat{m}_{+} \hat{m}_{-}\right), \quad \hat{m}_{ \pm}=m_{ \pm} / E .
$$

In the interaction region, $p^{0}$ does not vanish, but in the context of a single integral equation (Bethe-Salpeter equation) it cannot be treated as a dynamical variable. Instead, integrals involving $p^{0}$ are treated as perturbations on $\delta\left(p^{0}\right)$-integrals (section 7 ).

The space coordinate transformation of (53) is

$$
\boldsymbol{r}_{1}=\boldsymbol{R}+\hat{E}_{2} \boldsymbol{r}_{l}, \quad \boldsymbol{r}_{2}=\boldsymbol{R}-\hat{E}_{1} \boldsymbol{r}_{l},
$$

where $z_{l}$ is Lorentz contracted, $z_{l}=z / \gamma$. The nonrelativistic approximation yields the familiar $\hat{E}_{i}=m_{i} / m$. 


\section{VECTOR POTENTIAL AND BETHE LOGARITHM}

In the presence of a four-potential $A^{\mu}, p_{1}^{\mu}$ and $p_{2}^{\mu}$ are replaced by

$$
\pi_{1}^{\mu}=p_{1}^{\mu}+e A^{\mu}\left(x_{1}\right), \quad \pi_{2}^{\mu}=p_{2}^{\mu}-Z e A^{\mu}\left(x_{2}\right)
$$

The coefficients $\hat{E}_{1}$ and $\hat{E}_{2}$ of the transformation (53) to $\pi^{\mu}$ and $\Pi^{\mu}$ remain unchanged in a perturbative treatment of $A^{\mu}$. In the dipole approximation $\boldsymbol{A}\left(x_{1}\right)=\boldsymbol{A}\left(x_{2}\right)$, (56) leads to

$$
\boldsymbol{\pi}=\hat{E}_{2} \boldsymbol{\pi}_{1}-\hat{E}_{1} \boldsymbol{\pi}_{2}=\boldsymbol{p}+\operatorname{re} \boldsymbol{A},
$$

where $r e=r \cdot e$ is the dipole radiation charge [8],

$$
r=\frac{1}{2}(Z+1)-\frac{1}{2}(Z-1) \hat{m}_{+} \hat{m}_{-}=1+\frac{1}{2}(Z-1)\left(1-\hat{m}_{+} \hat{m}_{-}\right) .
$$

In the following, we use the Coulomb gauge in the cms and replace $\boldsymbol{p}$ by $\boldsymbol{\pi}$ in (2). To exhibit its $E^{2}$-dependence, we also multiply the equation by $E$ :

$$
\left(E \epsilon-E V-m_{1} m_{2} \beta\right) \psi=\gamma_{5}\left(\boldsymbol{\sigma}_{1}-i \boldsymbol{\sigma}^{\times} V / E\right) E \boldsymbol{\pi} \psi .
$$

The substitution

$$
\boldsymbol{r}=E \boldsymbol{\rho}, \quad \boldsymbol{p}=\boldsymbol{p}_{\rho} / E, \quad \boldsymbol{\pi}=\boldsymbol{\pi}_{\rho} / E
$$

brings (62) into a form which contains only even powers of $E$. For the moment, we assume $V=-Z \alpha / r, E V=-Z \alpha / \rho=V(\rho)$. Deviations will be discussed in section 7. For the Lambshift calculation, one neglects the hyperfine operator and obtains an explicit eigenvalue equation for $E \epsilon=\left(E^{2}-m_{1}^{2}-m_{2}^{2}\right) / 2$,

$$
h \psi=E \epsilon \psi, \quad h=m_{1} m_{2} \beta+V(\rho)+\boldsymbol{\alpha} \boldsymbol{\pi}_{\rho} .
$$

Its Coulomb Greens function $G$ satisfies the usual equation [2], taken in the new variables $\rho, \rho^{\prime}$ :

$$
\left[\nabla_{\rho}^{2}+(E \epsilon)^{2}-m_{1}^{2} m_{2}^{2}+2 E \epsilon Z \alpha / \rho+(Z \alpha+i \boldsymbol{\alpha} \hat{\boldsymbol{\rho}}) Z^{2} \alpha^{2} / \rho^{2}\right] G\left(\boldsymbol{\rho}, \boldsymbol{\rho}^{\prime}, E \epsilon\right)=\delta\left(\boldsymbol{\rho}-\boldsymbol{\rho}^{\prime}\right) .
$$

Evidently, it is also independent of the signs of $m_{1}$ and $m_{2}$. The same remark applies to $r$ (61), but that expression uses the low-energy dipole approximation. Apart from that, the Bethe logarithm can only depend on $E^{2}, m_{1}^{2}$ and $m_{2}^{2}$. Its proportionality to $r^{2}$ has been noted previously [14,15]. Inspection of the formulas collected in [2] reveals another

small s-state correction, which is also proportional to $r^{2}$. Moreover, both corrections are proportional to $m_{1} m_{2}$ and therefore pushed under the square root in (3), where they appear as quantum defects:

$$
\beta_{B}=\frac{4 \alpha^{3}}{3 \pi} r^{2} \ln k_{0}(n, l), \quad \beta^{\prime}=-\frac{4 \alpha^{3}}{3 \pi} r^{2}\left(\frac{5}{6}-\ln \alpha^{2}\right) .
$$

After the extraction of $\beta_{B}$ and $\beta^{\prime}$, there remains a somewhat reduced Salpeter shift, 


$$
\begin{aligned}
\Delta E_{S a l}^{\prime} & =-\frac{\mu_{n r}^{2} Z^{5} \alpha^{5}}{m \pi n^{3}}\left[\frac{7}{3} a_{n}^{\prime}+\frac{1}{m_{+} m_{-}} \delta_{l 0}\left(m_{2}^{2} \ln \frac{m^{2}}{m_{2}^{2}}-m_{1}^{2} \ln \frac{m^{2}}{m_{1}^{2}}\right)\right], \\
a_{n}^{\prime} & =-2 \delta_{l 0}\left[\ln \frac{2 \alpha}{n}+\sum_{i=1}^{n} \frac{1}{i}+\frac{1}{2}-\frac{1}{2 n}\right]+\frac{1-\delta_{l 0}}{l(l+1)(2 l+1)},
\end{aligned}
$$

with $a_{1}^{\prime}=-2 \ln (2 \alpha)-2$. The denominator $m_{+} m_{-}$in (67) will be discussed in the next section. The factor $1 / m$ in front disappears in the expression for $E^{2}$, as $E^{2} \approx m^{2}+2 m E_{b}$ according to (四).

\section{BETHE-SALPETER EQUATION, VACUUM POLARIZATION AND FORM FACTOR}

Continuing with the $T$-matrix of section 3, the two-photon exchange part $T^{(2)}$ may be used to derive corrections to the main interaction, including the Salpeter shift (67). However, here we consider instead the more commonly used Bethe-Salpeter equation. It is formulated in the cms, where $(53)$ reduces to

$$
p_{1}^{\mu}=p^{\mu}+E_{1} g_{\mu 0}, \quad p_{2}^{\mu}=-p^{\mu}+E_{2} g_{\mu 0},
$$

and $p^{\mu}$ is an integration variable. Suppressing the integration, the BS-equation applies to the two-fermion Greens function $G=G^{(16)}$

$$
G^{(16)}=S^{(16)}+S^{(16)} K^{(16)} G^{(16)},
$$

where $S^{(16)}$ is the product of two free fermion propagators in momentum space

$$
\begin{gathered}
S^{(16)}=\left(\not p_{1}-m_{1}\right)^{-1} \otimes\left(\not p_{2}-m_{2}\right)^{-1}=\left(\not p_{1}+m_{1}\right) \otimes\left(\not p_{2}+m_{2}\right) / D_{1} D_{2}, \\
D_{1}=p_{1}^{2}-m_{1}^{2}+i \varepsilon=p^{2}+2 E_{1} p^{0}+k^{2}+i \varepsilon, \quad D_{2}=p_{2}^{2}-m_{2}^{2}+i \varepsilon=p^{2}-2 E_{2} p^{0}+k^{2}+i \varepsilon,
\end{gathered}
$$

and the kernel $K^{(16)}$ is the sum of all irreducible Feynman diagrams. Our proposed eightcomponent formalism simplifies $S$ at the expense of $K$. Taking $K$ from the matrix elements $\chi^{\dagger} K \psi$ analogous to the $8 \times 8$ Matrix $M$ in (22), one has

$$
S^{(16)} K^{(16)}=S^{(8)} K^{(8)}, \quad S^{(8)}=\sum v_{L P} w_{L P}^{\dagger} / D_{1} D_{2}
$$

In a matrix notation where $\gamma^{0}=\beta$ and $\gamma_{5}$ are identical for particles 1 and 2, the spin summation is now

$$
\sum v_{L P} w_{L P}^{\dagger}=m_{2} E_{1}+m_{1} \beta E_{2}-m_{-} p^{0}-\gamma_{5} \boldsymbol{p}\left(m_{2} \boldsymbol{\sigma}_{1}-m_{1} \beta \boldsymbol{\sigma}_{2}\right) .
$$

It is only a first-order polynomial in $p_{1}, p_{2}$. Consequently, the numerator of $S^{(8)}$ is linear in the integration variables, whereas $S^{(16)}$ is quadratic. One may in fact go one step further and use irreducible representations of the Lorentz group, in which case one arrives at $S^{(4)}=$ 
$1 / D_{1} D_{2}$. This amounts to the elimination of the dotted spinor components from $\psi$ [16]; it is widely used in QCD calculations [17]. Four bound states, however, it has the disadvantage of suppressing explicit parity invariance.

The last bracket in (74) is $\left(m_{-} \boldsymbol{\sigma}+m_{+} \Delta \boldsymbol{\sigma}\right) / 2$ in the notation (18), and $m_{-} \boldsymbol{\sigma}$ may be converted into $m_{+} \boldsymbol{\sigma}$ by the transformation (20)

$$
v_{L P}=c v, \quad w_{L P}=c^{-1} w, \quad \sum v w^{\dagger}=\left(\epsilon-\gamma_{5} \boldsymbol{\sigma}_{1} \boldsymbol{p}+\mu \beta+p^{0} m_{-} / m_{+}\right) m_{+} .
$$

To handle the $p^{0}$-integration, one may use the formula $1 /(x-i \varepsilon)=P / x+i \pi \delta(x)(P=$ principal value):

$$
2 E / D_{1} D_{2}=2 i \pi \delta\left(p^{0}\right) /\left(\boldsymbol{p}^{2}-k^{2}\right)-1 / D_{1}\left(p^{0}+i \varepsilon\right)+1 / D_{2}\left(p^{0}-i \varepsilon\right),
$$

and treat all $p^{0}$-integrals except the first one as perturbations of the Greens function. In these integrals, the spin summation (75) reduces to $\sum v w^{\dagger} / m_{+}=\epsilon-\boldsymbol{\alpha} \boldsymbol{p}+\mu \beta$, which is simply the expression for a single-particle spin summation. In the remaining integrals, the complete two-fermion propagator has the more explicit numerator

$$
2 E \sum v w^{\dagger} / m_{+}=E^{2}-\left(m_{1}^{2}+m_{2}^{2}-2 m_{1} m_{2} \beta\right)\left(1-E p^{0} / m_{+} m_{-}\right)-2 \gamma_{5} \boldsymbol{p}_{\rho} \boldsymbol{\sigma}_{1} .
$$

After integration, this $p^{0}$-dependence produces factors $\left(m_{1}^{2}+m_{2}^{2}\right) / m_{+} m_{-}$and $m_{1} m_{2} \beta / m_{+} m_{-}$. Such factors may also arise from the denominators $1 / D_{1} D_{2}^{X}$ of the crossed graph, which contain the combination $p^{0}\left(E_{1}-E_{2}\right)=E p^{0} \hat{m}_{+} \hat{m}_{-}$. To check the mass dependence in the $\delta_{l 0}$-piece of $\Delta E_{\text {Sal }}^{\prime}$ (67), notice $m_{2}^{2} \ln \left(m^{2} / m_{2}^{2}\right)-m_{1}^{2} \ln \left(m^{2} / m_{1}^{2}\right)=$ $m_{+} m_{-} \ln \left(m_{1} m_{2} / m^{2}\right)+\left(m_{1}^{2}+m_{2}^{2}\right) \ln \left(m^{2} / m_{1} m_{2}\right)$.

We conclude with a discussion of the potential $E V(r) \rightarrow V(\rho)$ in the presence of vacuum polarization and nuclear charge distribution. For a heavy particle $1\left(\mu^{-}\right.$or $\left.\bar{p}\right)$, electronic vacuum polarization is so large in low- $l$-states that it must be added to the Coulomb potential $V_{C}$, in the form of the Uehling potential:

$$
V=V_{C}+V_{U}, \quad V_{U}=-\frac{Z \alpha^{2}}{3 \pi r} \int_{0}^{\infty} d \lambda^{2} e^{-\lambda r} S\left(\lambda^{2}\right),
$$

with $S_{e}=\left(1-4 m_{e}^{2} / \lambda^{2}\right)^{1 / 2}\left(1+2 m_{e}^{2} / \lambda^{2}\right) / \lambda^{2} \Theta\left(4 m_{e}^{2}\right)$ in the electronic part of $V_{U}$. At fixed $\lambda$, the $r$-dependence is easily converted to a $\rho$-dependence, $(E / r) e^{-\lambda r}=\rho^{-1} e^{-\lambda E \rho}$ :

$$
V_{U}(\rho)=-\frac{Z \alpha^{2}}{3 \pi \rho} \int_{0}^{\infty} d \lambda^{2} \mathrm{e}^{-\lambda E \rho} S\left(\lambda^{2}\right)
$$

The substitution $\lambda E=\lambda^{\prime}$ shows that the integral depends only on $E^{2}$. There is also a second-order recoil correction $V_{U r}^{(2)}$ which is spin-independent [18]. For antiprotonic atoms, the radius of the vacuum polarization cloud is much larger than the Bohr radius $(Z \alpha \mu)^{-1}$ : below a critical value $l_{c}$ of the orbital angular momentum $l$, vacuum polarization exceeds all relativistic effects (protonium $\bar{p} p$ and $\bar{p}^{3} \mathrm{He}$ have $l_{c}=3$ and 7 , respectively), for all values of $n$ [19]. For these orbitals, $V$ is best constructed numerically from (79), particularly for the calculation of annihilation which is localized at very small $\rho$. However, for the calculation of the fine and hyperfine structure of these inner orbitals, the form ([9) suggests the introduction of a running electric coupling constant $\alpha_{e}$ : 


$$
V=V_{e}+\delta V_{U}, \quad V_{e}=-Z \alpha_{e} / \rho, \quad \delta V_{U}=V_{U}+Z\left(\alpha_{e}-\alpha\right) / \rho, \quad\left\langle\delta V_{U}\right\rangle=0 .
$$

In this manner, the nonperturbative result (3) remains applicable, with $\alpha_{e}>\alpha$.

Recoil corrections to an extended nuclear charge distribution $\rho_{c}(r)$ are particularly large for muonic atoms.

$$
V(\rho)=-Z \alpha \int d^{3} r|\boldsymbol{\rho}-\boldsymbol{r} / E|^{-1} \rho_{c}(r), \quad \rho_{c}(r)=\int d^{3} q \mathrm{e}^{-i \boldsymbol{q} \boldsymbol{r}} F\left(q^{2}\right) .
$$

Again, $V(\rho)$ is $E^{2}$-dependent.

\section{ANGULAR MOMENTUM DEFECTS AND BARKER-GLOVER TERM}

To order $\alpha_{Z}^{2}=Z^{2} \alpha^{2}, \delta l$ is easily found for particles of arbitrary masses $m_{i}$ and $g$-factors $g_{i}=2\left(1+\kappa_{i}\right) . \delta l$ is conveniently expressed as follows:

$$
\delta l / \alpha_{Z}^{2}=-(1+a) /(2 l+1) .
$$

With the abbreviations $\hat{m}_{i}=m_{i} / E, \hat{\epsilon}=\epsilon / E$ and the combinations

$$
\begin{aligned}
& c_{1}^{2}=1-\hat{m}_{1}^{2}-\hat{\epsilon}+2 \kappa_{1} \hat{m}_{2}, \quad c_{2}^{2}=1-\hat{m}_{2}^{2}-\hat{\epsilon}+2 \kappa_{2} \hat{m}_{1}, \\
& c^{2}=1+2 \kappa_{1} \hat{m}_{2}+2 \kappa_{2} \hat{m}_{1}+2 \hat{\epsilon}\left(1-g_{1} g_{2} / 4\right),
\end{aligned}
$$

one finds

$$
a(l=f \pm 1)= \pm c^{2} /(2 j+1) \pm g_{1} g_{2} \hat{\epsilon} /(4 f+2)
$$

(where a sign error of [9] for $l=f+1$ is corrected), and

$a(l=f)= \pm \frac{c^{2}}{4 F^{2}}\left[\sqrt{(2 f+1)^{2}-16 F^{2} \frac{c_{1}^{2} c_{2}^{2}}{c^{4}}} \mp 1\right] \approx \pm \frac{c^{2}}{2 j+1} \mp \frac{2 c_{1}^{2} c_{2}^{2}}{c^{2}(2 f+1)}\left[1+\frac{4 F^{2} c_{1}^{2} c_{2}^{2}}{c^{4}(2 f+1)^{2}}\right]$.

In (85), $l=f \pm 1$ implies $j=j_{1}=f \pm \frac{1}{2}=l \mp \frac{1}{2}$. For $g_{1}=g_{2}=2, c=1$ leads exactly to the Dirac fine structure component (43) of $\delta l$, for all values of $m_{1}$ and $m_{2}$, including $m_{1}=m_{2}$ as in positronium. The case $l=f$ is more complicated, because the two values $j=l+\frac{1}{2}$ and $j=l-\frac{1}{2}$ are mixed by the hyperfine interaction. We therefore define $j$ as that value in the integer $2 j+1$ which appears in the main term $c^{2} /(2 j+1)$ of $a(l=f)$ after expansion of the square root. It is frequently said that the fine structure contains the "Barker-Glover" term (for $l>0$ ) [2, [],

$$
E_{B G}=\alpha_{Z}^{4} \mu^{3}(l-j) /\left[(2 j+1)(2 l+1) m^{2} n^{3}\right] .
$$

This is true for a spinless nucleus, except that the electron's anomalous magnetic moment frequently reverses the effect [11. On the other hand, the weighted average over the hyperfine structure produces a more complicated expression,

$$
\bar{a}=(l-j)\left[2 c^{2}+g_{1} g_{2} \hat{\epsilon} / 2-2\left(c_{1}^{2} c_{2}^{2} / c^{2}\right)\left(1+4 L^{2} c_{1}^{2} c_{2}^{2} / c^{4}(2 l+1)^{2}\right)\right] /(2 j+1) .
$$


This is the final result, and as a rule it is much larger than the Barker-Glover term. For $g_{1}=g_{2}=2$, however, $c_{1}^{2} c_{2}^{2} \approx \mu / m \approx \hat{\epsilon}$ to this order in $\alpha$, and the main terms cancel in (88). There remains a small rest,

$$
\Delta \bar{E}=\alpha_{Z}^{2} \mu_{n r} \bar{\delta} l /(2 l+1) n^{3}=-\mu_{n r} \alpha_{Z}^{4} \bar{a} / n^{3}=4 \mu_{n r}^{3} \alpha_{Z}^{4}(l-j) L^{2} /(2 l+1)^{3}(2 j+1) m^{2} n^{3},
$$

which replaces $E_{B G}$ in the case of muonium.

ACKNowledgments: The authors would like to thank S. Karshenboim and A. Yelkhovsky for helpful comments. This work has been supported by the Deutsche Forschungsgemeinschaft. 


\section{REFERENCES}

[1] H. Grotch and D.R. Yennie, Rev. Mod. Phys. 41, 350 (1969)

[2] J.R. Sapirstein and D. Yennie, in: Quantum Electrodynamics (World Scientific, Singapore, 1990)

[3] K. Pachucki et al, J. Phys. B 29, 177 (1996)

[4] A. Yelkhovsky, JETP 83, 230 (1996)

[5] W.E. Caswell and G.E. Lepage, Phys. Lett. B 167, 437 (1986)

[6] P. Labelle, S.M. Zebarjad and C.P. Burgess, Phys. Rev. D 56, 8053 (1997),

A.H. Hoang, P. Labelle and S.M. Zebarjad, Phys. Rev. Lett. 79, 3387 (1997)

[7] H. Pilkuhn, J. Phys. B 25, 289 (1992),

H. Pilkuhn and F. Stäudner, Phys. Letters A 178, 156 (1993)

[8] M. Malvetti and H. Pilkuhn, Phys. Rep. C 248, 1 (1994)

[9] H. Pilkuhn, J. Phys. B 28, 4421 (1995)

[10] E. Brezin, C. Itzykson and J. Zinn-Justin, Phys. Rev. D 1, 2349 (1970)

[11] H. Pilkuhn, J. Phys. B 17, 4061 (1984)

[12] S.N. Gupta, Quantum Electrodynamics (Gordon and Breach, New York 1977),

S.N. Gupta, W.W. Repko and C.J. Suchyta III, Phys. Rev. D 40, 4100 (1989)

[13] K. Pachucki and H. Grotch, Phys. Rev. A 51, 1854 (1995),

E.A. Golosov et al., JETP 80, 208 (1995)

[14] G.W. Erickson, J. Phys. Chem. Ref. Date 6, 833 (1977)

[15] S.G. Karshenboim, JETP 80, 593 (1995)

[16] L.M. Brown, Phys. Rev. 111, 957 (1958),

M. Tonin, Nuo. Cim. 14, 1108 (1959)

[17] G. Chalmers and W. Siegel, hep-ph/9708251

[18] J. Fröhlich and H. Pilkuhn, J. Phys. B 17, 147 (1984)

[19] S. Barmo, H. Pilkuhn and H.G. Schlaile, Z. Phys. A 301, 283 (1981) 